\title{
Application and Research of Integrated Information Exchange System Between Substation and Dispatch Center
}

\author{
Ning Jiang ${ }^{1}$, Lin $\mathrm{Li}^{1}$, Yao Wang ${ }^{1}$, Guodong $\mathrm{Li}^{1}$, Lingxu Guo ${ }^{2}$ and Jian Chen ${ }^{2}$ \\ ${ }^{1}$ Tianjin Electric Power Research Institute, State Grid, Tianjin, China \\ ${ }^{2}$ Tianjin Electric Dispatch and Control Center, State Grid, Tianjin, China
}

\begin{abstract}
This paper realizes direct communication between intelligent substation and dispatch center based on IEC61850 standard. An integrated information exchange system is established to achieve the above content. The communication uses Client/Server mode. An intelligent data gateway is used to integrate functions in substation. The server and client complete data read and write operations by real-time database in substation. The client in dispatch center is integrated in SCADA system. It collects data from the server in substations, and sends control and summons commands. The integrated information exchange system comprises IEC 61850 front-end software in dispatch center, integrated chart-module maintenance software, IEC 61850 telecontrol workstation software in substation and system configuration tool software. The system realizes data terminal maintenance, improve interoperability between substation and dispatch center, improve the ability of information exchange between substation and dispatch center.
\end{abstract}

Keywords-integrated communication; common information model; IEC 61850 model

\section{INTRODUCTION}

The model of Smart Substation and dispatch center are based on IEC61850 and IEC61970 standard respectively to modeling, maintenance and management. The communication between substation and dispatch center still uses the IEC 60870-5-101 or IEC $60870-5-104$ protocol. The traditional protocol does not support the object-oriented modeling method. The model based on IEC 61850 must be converted to information points. Thus the configuration of forwarding information is very complex. the workload of configuration and debugging is very hard and easy to make mistakes. At present, the development of intelligent scheduling is seriously restricted by the way of communication based on point table. The establishment of an integrated information exchange platform can solve the problem of low communication efficiency and incomplete mode in [1][2][3].

International standard of substation communication networks and systems is introduced in [4], including the structure and characteristics of IEC61850. The information in the smart substation is classified, and the data information flow in the communication network is analyzed in [5]. The data exchange experiment platform was designed by [6], and the feasibility of the interoperability between the dispatching system and the intelligent substation system was studied. A distributed network modeling architecture of substation dispatch center is designed and studied, and the scheme of the model is studied in [7]. An intelligent communication equipment based on IEC 61850 standard is proposed in [8], which improves the efficiency of information transmission and network performance. In literature [9], an information integration technology based on Protege is studied. The ontology model of SCL and CIM is constructed respectively, and the formal description of the two models is realized through OWL language. The mapping relationship between CIM model and SSD model is discussed, and the conversion technology between SSD model and CIM model is studied and implemented in literature [10].

\section{CONSTRUCTION SCHEME}

A. Communication Interface Scheme

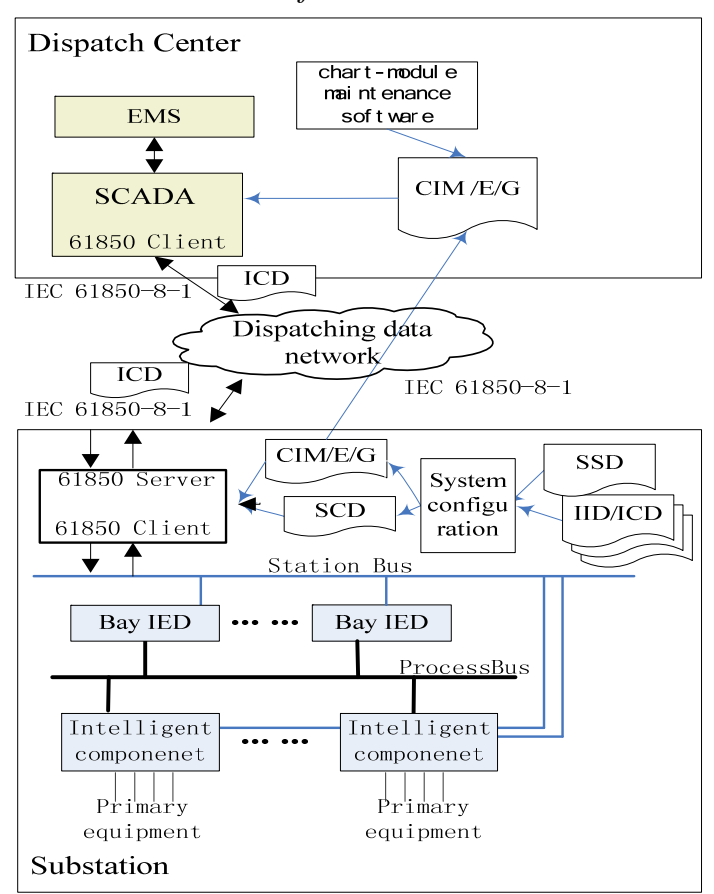

FIGURE I. COMMUNICATION INTERFACE SCHEME.

The communication architecture is extended from substation to control center. The communication uses 
Client/Server mode to achieve the direct communication based on IEC61850 standard. This mode eliminates the conversion from model to information point table. Unified communication architecture between substation and control center can enhance the efficiency and accuracy of the information exchange. Data from substation to dispatch center can be transported flexibly and securely.

Intelligent data gateway in transformer substation integrate functions such as disturbance recorder, Relay Protection information management, WAN control, fault location based on wide-area information, state estimation in substation.

The communication object model structure which based on IEC61850 is a kind of tree hierarchical structure. The substation communication models upload to dispatch center by configuration file. The model can be accessed online by services of read data attribute and read directory. The process of add communication model tree as shown in Figure II.

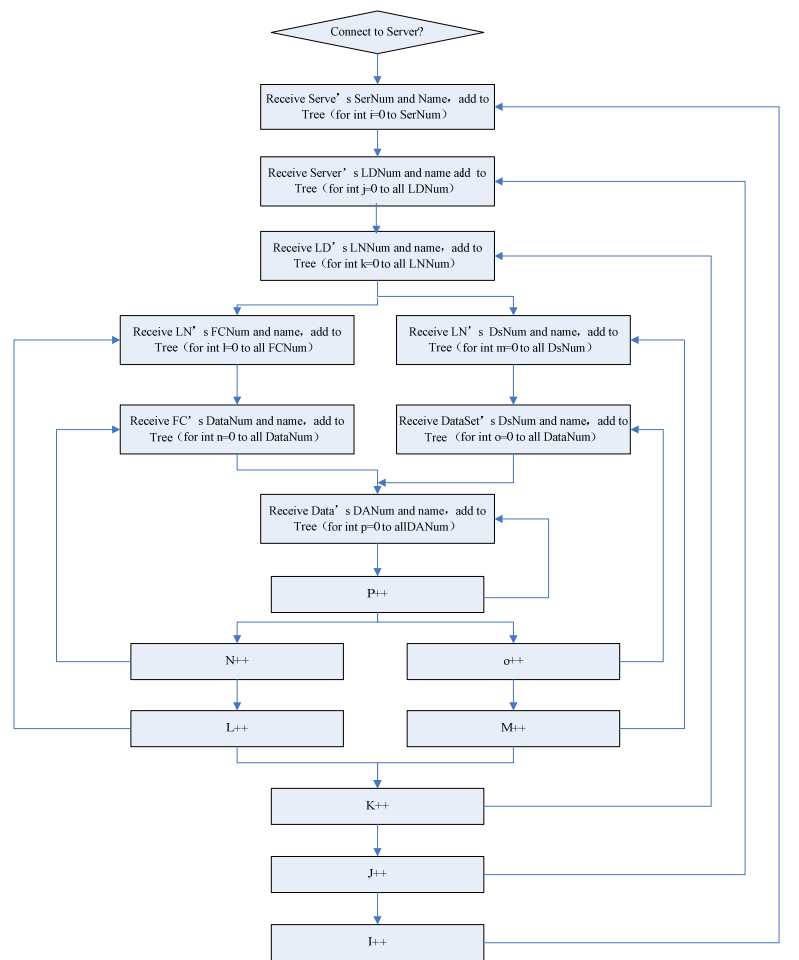

FIGURE II. FLOW CHART OF ADD 61850 COMMUNICATION MODEL TREE

Intelligent data gateway integrates IEC 61850 Client and Server. Client create information model based on DL/T 860 standard. Client gathers information from the substation, and sends control and summons commands to bay level equipment. Server create information model comply with dispatch center functional requirements, information exchange to IEC 61850 client at dispatch center. The server and client complete data read and write operations by real-time database. The 61850 model instance and the connection relation of them are established by parse CID/SCD file when the system is initialized. The process of add object properties as shown in Figure III.

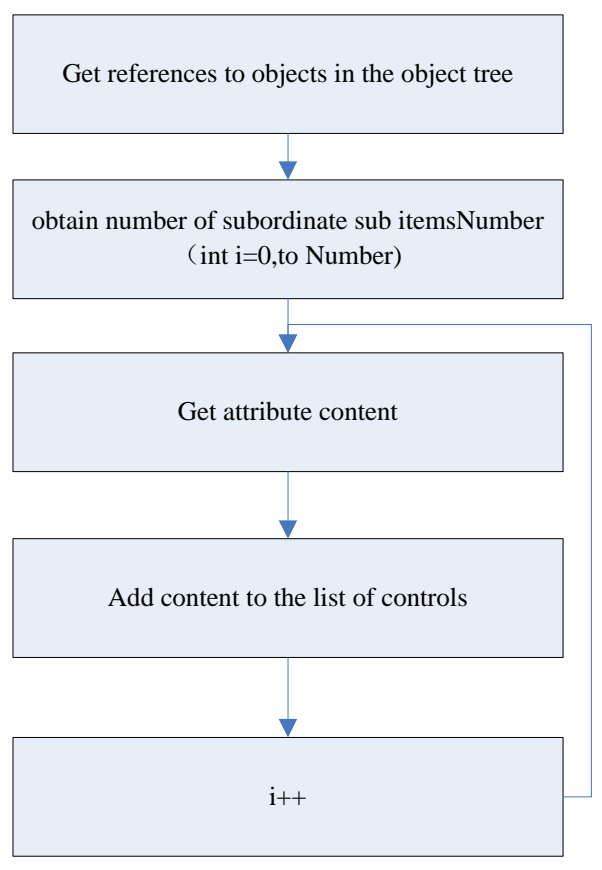

FIGURE III. Add Object Property

The process of log manages as shown in Figure IV.

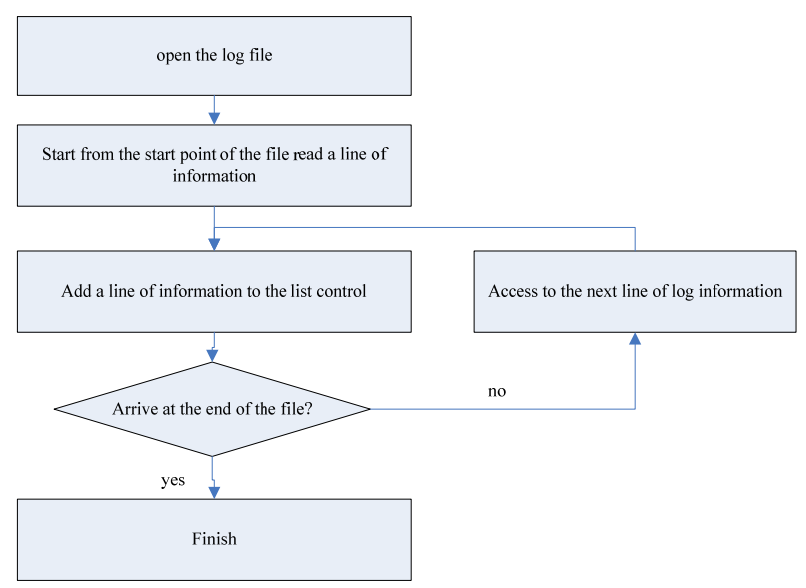

FIGURE IV. LOG MANAGEMENT

The IEC 61850 client in dispatch center is integrated in SCADA system. It collects data from the server in all substations, and sends control and summons commands. The 61850 model instance and the mapping relation between 61970 CIM model instance is established by parse unified data model file when the system is initialized. The client exchange information with data processing module by SCADA message bus. The client in dispatch center realizes model analysis based on IEC61850 standard. It also realizes chart-module and real-time data exchange which comprise such as control command and real time data update between intelligent data gateway in transformer substation 


\section{B. Real-time Data Transmission}

In substation the communication between bay level equipment and control level equipment is based on IEC 61850 standard. The communication between substation automation telecontrol system and dispatch is also based on IEC 61850 standard.

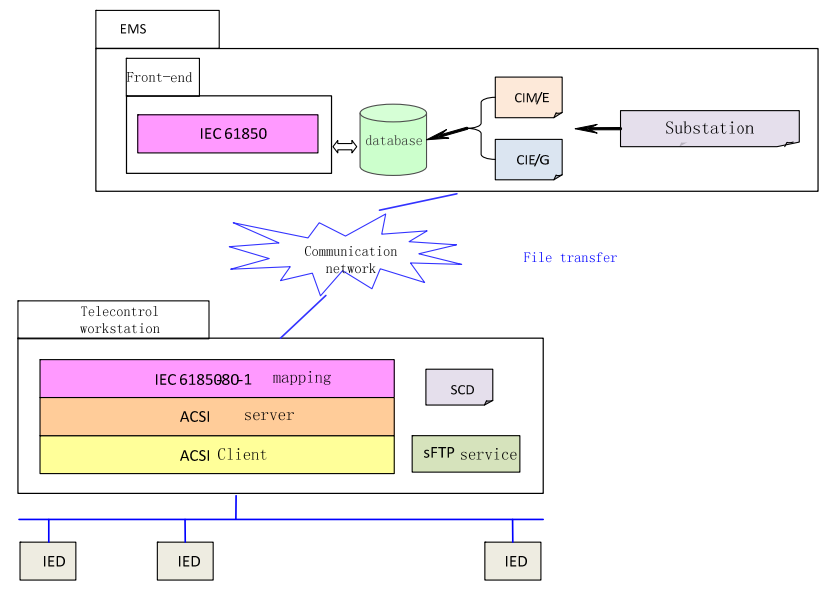

FIGURE V. STRUCTURE OF COMMUNICATION STACK

The data in intelligent data gateway server is not easily transmitted from intelligent data gateway client. The data in server is much more accurate and reliable by judge and calculate redundant data from client. These data can be used in substation advanced application such as state estimation.

Modeling principle of intelligent data gateway:

(1) The ICD is based on DL/T 860 standard in substation, include all of IED data in the substation.

(2) The communication between dispatch center and intelligent data gateway is based on IEC 61850 gateway pattern (multiple LD in single IED).

(3) The intelligent data gateway as a single IEC include all LN data and related synthetic data of the physical equipment.

The mapping relationship between 61850 model instance and 61970 model instance is established by IEC 61850 frontend software to parse unified data model file when the system is initialized.

The IEC 61850 client in dispatch center receive data from Server in substation and sends control and summons commands. The communication between substation and dispatch center uses MMS stack based on TCP/IP to enhance interoperability. The maintenance interface is used to check communication modeling and real-time data. Call real-time data service interface to write remote measure and remote communication to the database.

\section{IEC61850 Front-end Software in Dispatch Center}

IEC61850 front-end software in dispatch center is deployed in D5000 platform, include 61850 data acquisition module, and real-time database interface, and system management interface, and communication parameters configuration module, etc.

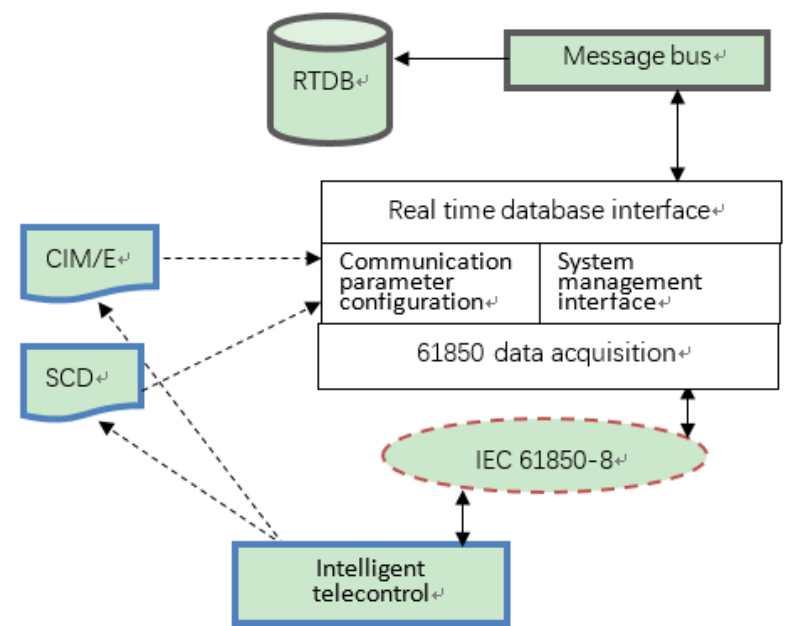

FIGURE VI. STRUCTURE OF IEC61850 FRONT-END SOFTWARE

Software is registered as a process of D5000 system through the system management interface. Its operation and exit is managed by the D5000 system.

The communication tree structure and the corresponding data mapping table is composed of the communicationconfigure management module of the software. The data is from the SCD and CIM/E files provided by the substation. Through the real time database interface module, the IEC 61850 data model and the D5000 data model are realized in the initialization.

D5000 real time database refresh through the IEC61850 client software to join the corresponding processing procedures, including the D5000 related Library. The location of database data, including access to the table name, domain number, number, station number and so on. A set of data access interface based on C99 standard is provided by the D5000 standard, and the internal interaction of data is realized through message bus.

Parse the CIM/E file and call the real-time database interface function when the software is initialization, associated 61850 references with database dots and then saved to the configuration file based on the only pathname; After receiving the data schlep by 61850 quote from the data gateway, send the information to SCADA by message bus interface. 


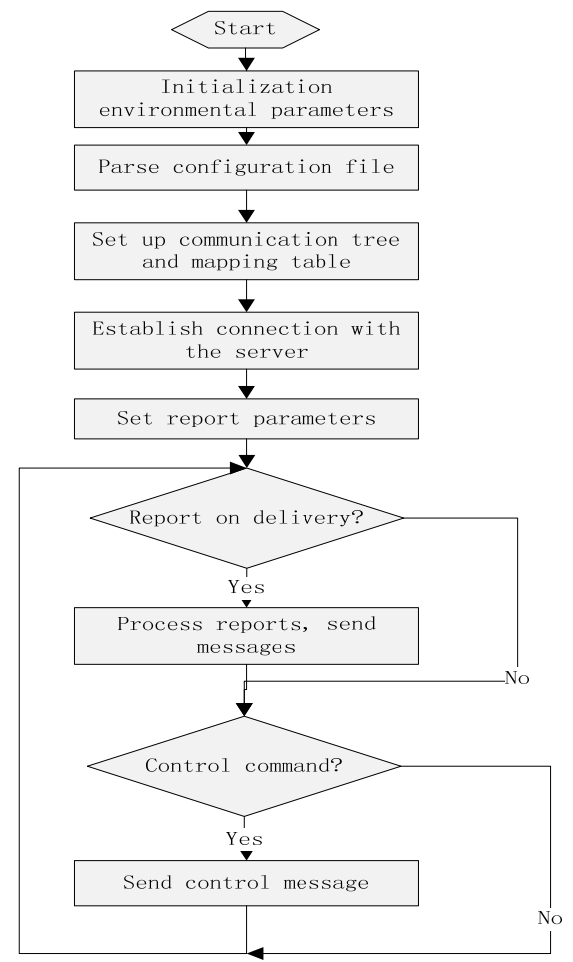

FIGURE VII. COMMUNICATION PROCESSING FLOW CHART

\section{Integrated Chart-module Maintenance Software}

Integrated chart-module maintenance software is mainly composed of SCD model management, CIM/E model management, SVG and CIM/G graphics management, model assembly and other modules.

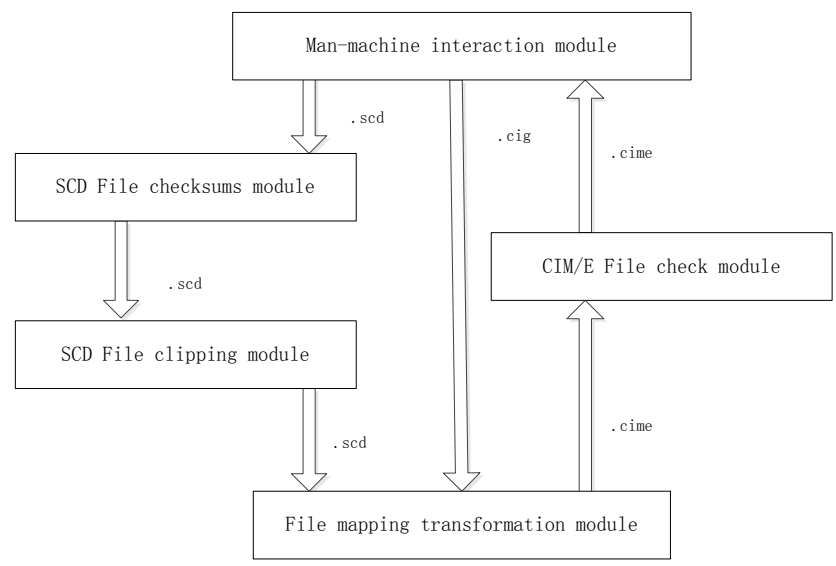

FIGURE VIII. FUNCTION OF SCD AND CIM/E FILE CONVERSION

The man-machine interface module's function is interacting with the user, provide the source file and rule file to SCD file check module and file conversion module; the SCD file clipping module and file mapping conversion module's function must be executed after received the return of verification success from the SCD file verification module; CIM /E file check module's function must be executed after received the return of conversion success from file mapping transformation module.

SCD file legitimacy verification, including the verification format, syntax and basic function description. Verification format is to check whether the file is in the correct format XML file. Syntax checking is to Check whether the documents are in compliance with the schema syntax rules defined in 61850-6 IEC. Check whether the data reference (such as attribute) of data-set and the report control block description is existed.

\section{THE INTEGRATED INFORMATION EXCHANGE SYSTEM BETWEen POWER TRANSFORMER SUBSTATION AND CONTROL CENTER}

\section{A. Architectural Structure}

The integrated information exchange system comprises IEC 61850 front-end software in dispatch center, integrated chart-module maintenance software, IEC 61850 telecontrol workstation software in substation and system configuration tool software.

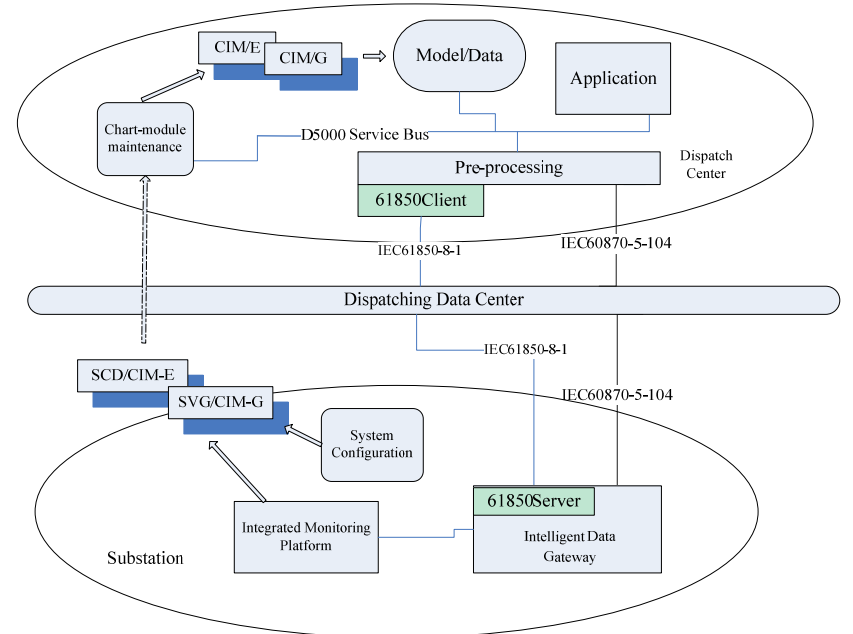

FIGURE IX. STRUCTURE OF INTEGRATED INFORMATION EXCHANGE SYSTEM

The system meets the demand of extendibility, reliability, standardization and maintainability. The system can realize data terminal maintenance, improve interoperability between substation and dispatch center, improve the ability of information exchange between substation and dispatch center.

\section{B. System Function}

(1) Real-time data exchange based on IEC 61850 standard: the communication between substation and dispatch center based on IEC 61850, support services such as association, directory, data reading, report, data set, control, setting and so on.

(2) Data terminal maintenance: system configuration tool in substation export SCD or CIM/E (include network model and measurement model) modeling file, SVG or CIM/G (include the association between CIM/E) graphic file; The 
integrated chart-module maintenance tool in dispatch center import, check, decode, combine and store SCD or CIM/E file to provide linkage of real time measurement for subsequent application.

(3) Remote measurement, remote signal and remote control.

(4) System in substation provide SCD file which include the total station information (contains SSD).

(5) The model of intelligent data gateway is independent IED. The IED will reduce data according to dispatch center's demand. The gateway is as the server to communicate with dispatch center.

(6) The pre software of com in dispatch center integrate IEC 61850 client which communicate with substation.

(7) The system provides Boundary maintenance, Model file management, Graphic information autochangeover functions.

\section{ENGINEERING APPLICATION}

\section{A. Main Work Contents}

Deploy Software integrated information exchange system between power transformer substation and control center.

Implementation principle: Minimize the impact of the online operating system.

The dispatch center includes: IEC 61850 front-end machine software, integrated chart-module maintenance software (include model import, model export, model check, model compare, boundary setting, model merging and so on).

The substation includes: intelligent data gateway (include intelligent substation IEC 61850 telecontrol workstation software), intelligent substation system configuration tool software.

\section{B. System Environment Requirements}

ZhongChuandong intelligent substation is chosen to deploy the system; In dispatch center the system is deploy at model center in SCADA and pre node.

Environment requirements of SCADA node: software version of pre software is V3.1, Service Bus and Message bus, real-time database, relational database; SCADA and manmachine console is available; The model is consistent with the operating system, ID of graphics and model is correct.

\section{Communication Channel Between Dispatch Center and Substation}

Add tj61850com node at tjscd1 node in model center, access dispatching data network and assign IP. Add an intelligent data gateway at substation, access dispatching data network and assign IP.

\section{Dispatch center}

The integrated information exchange system is deployed in model center which is located at Tianjin dispatch center. integrated chart-module maintenance software is deployed at tjscd1 node. IEC 61850 front-end software is deployed at tj61850com node.

\section{Application program:}

(1) IEC 61850 front-end software in dispatch center source program: \$HOME/src/IEDExplorer dynamic library: \$HOME/src/bin/IEDExplorer /lib executable program: \$HOME/src/bin/IEDExplorer configuration file: \$HOME/src/bin/IEDExplorer /IEDExplorerfiles environment variable: setenv QTDIR / opt/ qtsdk-2010.01 /qt

setenv QMAKESPEC / opt/ qtsdk-2010.01 /qt/ mkspecs/ linux-g++

setenv QMAKE_CONFIG_PATH /opt/ qtsdk-2010.01/ qt/lib

setenv APCHOME \$D5000_HOME/src/bin/IEDExplorer setenv PATH /opt/qtsdk-2010.01/qt/bin:\$APCHOME/bin/: \$PATH

setenv LD_LIBRARY_PATH /opt/ qtsdk-2010.01/ qt/ lib: \$APCHOME/lib: \$LD_LIBRARY_PATH

setenv LIBPATH/ opt/ qtsdk-2010.01/ qt/ lib: \$ACSIHOME/: \$APCHOME/lib: \$D5000_HOME/lib: \$LIBPATH

(2) Integrated chart-module maintenance software: Do not change the existing model configuration, executable programs, libraries, to ensure that the west is not affected by the splicing function model. Add a new program and configure the other path / file name.

source code: \$HOME/src/app

Background script: \$HOME/src/bin/

Background executable program: \$HOME/src/bin/

Foreground script: \$HOME/bin/mmiexec

Foreground executable program: \$HOME/ bin/ mmiexec/ jars/

(3) Model, Graphic and Configuration

Model file: \$HOME/data/cimdata

Graphic file: \$HOME/data/graph

Model configuration file: \$HOME/data/cimdata/CF

Pre configuration file: \$HOME/data/modify_meas, \$HOME/data/publish_meas

\section{E. Substation}

(1) station to add 1 RTU (data communication network, IEC61850 server shutdown) software configuration to control center.

(2) 1 Independent channel (channel data network) model system to small center location is located in Tianjin city the main room.

(3) to the original ship station is on the basis of the data according to the established model and test specification, SCD, CIM/E, CIM/G file. Automatically export the picture on the 
point to CIM/E, in addition to traditional telemetry and remote information, to protect the monitoring information, the two device monitoring information (soft board, etc.) in the picture to do all, export to CIM/E, CIM/G. CIM/G including the main wiring diagram, the diagram of 3 voltage level, the interval diagram.

\section{CONCLUSION}

This paper analyzes the communication mode and technology between substation and dispatch center, gives the concrete implementation method of unified information exchange. The source maintenance and the whole network sharing of the information model are realized. Research and development of intelligent substation and control center integrated information interaction system based on IEC61850 and IEC61970 standard. The remote communication between dispatch center and substation is collecting and delivery straightly based on IEC61850 standard, improve interoperability of communication substation dispatch center.

\section{REFERENCES}

[1] Huang Haifeng, Cao Yang, Song XinPower. Model Management System Fitted for Smart Dispatch. 2009Chin.Soe.for Elc.Eng, VOI.29Supplement,pp.7-10.

[2] Tan Wenshu. An Introduction to Substation Communication Network and System-IEC 61850. Power System Technology.,2001, 25 (9) :pp.8-11

[3] Xue Chen, Li Canbing, Huang Xiaoqing. Application of Intelligent Substation Information Integration. Electric Power Automation Equipment, 2011, 31(7):pp.110-114.

[4] Zhu Botong, Cheng Zhihai, Tang Zhiqiang. Interoperate Research of the Intelligent Substation and Dispatching Center Based on CIM Model. Power System Protection and Control.2014,41(10) :pp.93-97

[5] Wang Jing, Sun Hongbin, Chen Runze. Two Stage Distributed Network Modeling Between Dispatch Center and Substation. Automation of Electric Power Systems. 2014,38(9):pp.107-113

[6] Wang Qiang, Xu Xiaolan, Ge Guangsheng. Key Technologies of Special Communication Device in Smart Substation. Power System Protection and Control, 2014,42(7):pp150-153

[7] Huang Song, Zhang Peichao, Li Can. Smart Grid Information Integration Technology Based on Ontology Mapping and Rule Reasoning. Power System Protection and Control,2012,40(20):pp.150155

[8] Wang Huapeng, Yang Wei, Xu Zhi. Research on Model Conversion Technology of Source Servicing in Unified Information Platform of Smart Substation. Electric Power Construction,2012,33(1):pp.27-31

[9] LIN Jinghuai, MI Weimin, YE Fei. Several Issues in Modeling Technique for Intelligent Dispatching[J]. Power System Technology, 2011,35(6):pp.1-4

[10] JU Yang. Modes of Web communication of digital substation[J]. Power System Protection and Control,2010,38(1):pp.92-95 